Research Article

\title{
UM ESTUDO DE CLIMATOLOGIA DIÁRIA DA TEMPERATURA MÍNIMA, MÁXIMA E CHUVA ACUMULADA E UMA APLICAÇÃO DE "MODEL OUTPUT STATISTICS" (MOS) PARA A PREVISÃO DE CURTO PRAZO NO ESTADO DO PARANÁ
}

\author{
Ilya S. Kim, Cesar A. Beneti \& Dornelles Vissotto Junior \\ Received November 08, 2001 / Accepted December 14, 2002
}

\begin{abstract}
Foi feito um estudo de climatologia das anomalias diária da temperatura mínima, máxima e chuva acumulada com uso de dados das estações meteorológicas da rede do Instituto Agronômico do Paraná. Foi mostrado que a distribuição de freqüência de ocorrência das anomalias de temperatura mínima é quase normal, mas a distribuição de freqüência de ocorrência das anomalias de temperatura máxima é quase uniforme e a distribuição de freqüência de chuva tem uma assimetria positiva. Foi adaptado o MOS (Model Output Statistics), onde foram usados a saída do modelo numérico do National Center for Environmental Prediction (NCEP - EUA) do período de abril de 1997 a março de 2000 e os dados correspondentes de temperatura mínima, máxima e chuva acumulada de 26 estações do IAPAR. Na implementação do MOS foi aplicado o método de grupos análogos. O MOS foi implementado separadamente para os períodos abril-setembro (período do ano frio) e outubromarço (período do ano quente). Foram calculadas e avaliadas as previsões com a utilização do método de validação cruzada ("cross validation") de 1 a 4 dias para o período de abril de 1997 à março de 2000 . A ocorrência de chuva é prevista com acerto de 78\%, 76\%, 75\% e 71\% no inverno e $72 \%, 71 \%, 68 \%$ e $67 \%$ no verão para 1 a 4 dias, respectivamente. O erro médio absoluto das previsões da temperatura mínima são 1,8, 1,9,2,1 e 2,2 no inverno e 1,2, 1,3,1,4 e 1,4 no verão para 1 a 4 dias, respectivamente. O erro médio absoluto das previsões da temperatura máxima são 2,1, 2,4, 2,5 e 2,7 no inverno e 2,0, 2,2,2,2 e 2,4 no verão para 1 a 4 dias, respectivamente. A avaliação das previsões mostrou uma boa vantagem das previsões do MOS diante das previsões de persistência e climatologia.
\end{abstract}

Palavras-Chave: Mos; Climatologia; Previsão estatística; Temperatura mínima e máxima; Chuva acumulada; Estado do Paraná.

\begin{abstract}
A STUDY OF DAILY CLIMATOLOGY OF MINIMUM AND MAXIMUM TEMPERATURE AND ACCUMULATED PRECIPITATION AND AN APPLICATION OF MODEL OUTPUT STATISTICS (MOS) FOR THE SHORT-TERM FORECASTS IN THE STATE OF PARANA' - Daily climatology of minimum and maximum temperature anomalies ( $\Delta T_{\text {min' }}$ $\Delta T_{\max }$ ) and also accumulated precipitation were studied with data from the Instituto Agronômico do Paraná (IAPAR)'s meteorological stations. It was shown that frequency distribution of $\Delta T_{\min }$ occurrences for all stations is almost normal, whereas frequency distribution of $\Delta T_{\text {max }}$ occurrences is almost uniform and frequency distribution of rainfall occurrences has positive symmetry. A Model Output Statistics (MOS) was adapted using data from National Center for Environmental Prediction (NCEP) numerical model outputs and from 26 IAPAR stations and with information of minimum and maximum temperatures and accumulated precipitation. In the MOS implementation it was utilized an analogs group method. MOS was implemented for periods of april-september (colder period) and october-march (warmer period). Forecasts were calculated and evaluated using a cross validation method for up to 4 days for the period of April 1997 to March 2000. The rainfall occurrences were predicted correctly $78 \%, 76 \%, 75 \%, 71 \%$ for 1 to 4 days, respectively,
\end{abstract}


during the winter, and 72\%, 71\%, 68\%, 67\% for 1 to 4 days, respectively, during the summer. Mean absolute errors for minimum temperature are 1.8, 1.9, 2.1 and 2.2 for 1 to 4 days, respectively, during the winter, and 1.2, 1.3, 1.4, 1.4 for 1 to 4 days, respectively, during the summer. Maximum temperature is predicted with mean absolute errors of 2.1, 2.4, 2.5, 2.7 for 1 to 4 days, respectively, during the winter, and 2.0, 2.2, 2.2, 2.4 for 1 to 4 days, respectively, during the summer. Comparisons of MOS forecasts with persistence and climatology for the same period showed the advantage of using MOS forecasts.

Keywords: Mos; Climatology; Statistical forecast; Minimum and maximum temperature; Accumulated precipitation.

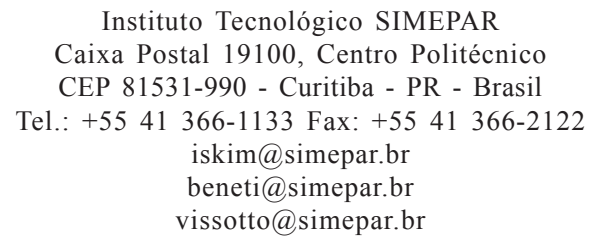

\section{INTRODUÇÃO}

Como é sabido, a saída do modelo numérico é uma previsão dos campos das variáveis meteorológicas em diferentes níveis numa grade específica. Nestas previsões existem erros sistemáticos que não dependem somente das falhas de parametrização física, mas dependem também da estação do ano, da localização da estação relativa à sub-grade do modelo, da topografia local ou do clima específico de diferentes partes nas grandes cidades que o modelo numérico não pode levar em conta. $\mathrm{Na}$ prática, é preciso ter previsões das variáveis e de fenômenos que não são normalmente resultados de modelos numéricos de tempo e para locais ou pontos, como por exemplo, numa estação meteorológica ou cidade. Em geral, os meteorologistas interpretam as saídas dos modelos numéricos com o uso da própria experiência adquirida e desenvolvem previsões das variáveis e fenômenos atmosféricos necessários. A qualidade destas previsões depende muito da experiência do meteorologista. Porém, existe outra possibilidade, que é a interpretação estatística da saída do modelo numérico, denominada MOS ("Model Output Statistics").

O MOS estabelece relações entre as previsões do modelo numérico como preditores e as observações como preditantes. A deficiência principal deste método é requerer uma base grande de dados das saídas do modelo numérico. Sabe-se que os modelos são continuamente modificados, e a obtenção destes dados é muito difícil. As relações estatísticas obtidas com as saídas do modelo anterior tornam-se não representativas. No entanto, alguns estudos mostram que pequenas alterações nos modelos numéricos não degradam substancialmente as equações do MOS (Erickson et al. (1991)).

Através do MOS foram desenvolvidas previsões de diversas variáveis, tais como: temperatura mínima e máxima, probalidade de ocorrência de precipitação, temperatura do ponto de orvalho, direção e velocidade do vento, ocorrência de tempestades (severas), altura da base de nuvens, visibilidade, temperatura horária, nevoeiro e outras variáveis meteorológicas (Jacks et al. (1989); Wilks (1990); Esterle (1992); Vislocky \& Fritsch (1995); Vislocky \& Fritsch (1997)). O MOS tem sido implementado em vários centros de meteorologia (Esterle (1992); Vasiliev (1991); Francis et al. (1982); Lemcke \& Kruizinga (1988); Conte et al. (1980); Tapp et al. (1986); Lu (1991); Azcarraga \& Ballester (1991); Brunet et al. (1988); Carter et al. (1989)). No Brasil também foi feito um experimento (Kim et al. (1998a) aplicando o MOS para previsão da temperatura mínima no inverno para as regiões Sul e Sudeste do Brasil com a saída do modelo numérico global CPTEC/COLA do Centro de Previsão e Estudos Climáticos do Instituto Nacional de Pesquisas Espaciais . Os resultados deste trabalho verificaram a possibilidade de aplicar o MOS na zona tropical e subtropical.

No presente trabalho foi aplicado o MOS para a previsão das temperaturas mínima e máxima e ocorrência e quantidade de chuva nas estações meteorológicas do estado do Paraná, para os períodos de 24, 48, 72 e 96 horas. No estudo foi 
aplicado o método de grupos análogos com o uso da saída do modelo numérico do Centro Nacional de Previsão Ambiental (NCEP) dos Estados Unidos do América.

\section{METODOLOGIA}

\section{Dados}

Como preditantes foram utilizados os dados de temperatura mínima $\left(T_{\min }\right)$, máxima $\left(T_{\max }\right)$ e chuva acumulada diária do período de abril de 1997 até março de 2000 das estações meteorológicas do Instituto Agronômico do Paraná (IAPAR). A localização das estações é mostrada na Fig. 1.

Como preditores foram utilizadas as saídas do modelo numérico global do NCEP para $36 \mathrm{~h}, 60 \mathrm{~h}$, $84 \mathrm{~h}$ e $108 \mathrm{~h}$ em pontos de grade geográfica com resolução de $2.5 \times 2.5$ graus, na área de $60^{\circ} \mathrm{W}$ à $45^{\circ} \mathrm{W}$ e $35^{\circ} \mathrm{S}$ à $17.5^{\circ} \mathrm{S}$, que compreende um setor sul do continente sul-americano e oceanos adjacentes (Fig. 2). A diferença entre o horário GMT e o horário local para o Paraná (Brasil) é de -3 horas, ou seja, a previsão para 36 horas do modelo NCEP representa 9 horas da manhã local do outro dia para o Brasil e assim por diante.

Foram selecionadas as seguintes variáveis da saída do modelo numérico: pressão ao nível médio do mar, precipitação acumulada em 24 horas na superfície, água precipitável instantânea, radiação de onda longa emergente no topo da atmosfera, umidade especifica e cobertura de nuvens. Nos níveis de pressão padrões de 1000, 925, 850, 700, 500, 400 e $300 \mathrm{hPa}$ foram também selecionadas as seguintes variáveis: geopotencial, temperatura do ar, umidade relativa, vento zonal e meridional e o análogo da velocidade vertical "ômega". Com uso dos dados de saída do modelo foram calculados os campos de gradientes zonais e meridionais das variáveis os quais também foram aplicados como preditores.

\section{Método de grupos análogos}

Para a implementação do MOS foi utilizado o método de análogos, com parâmetro de semelhança $S:$

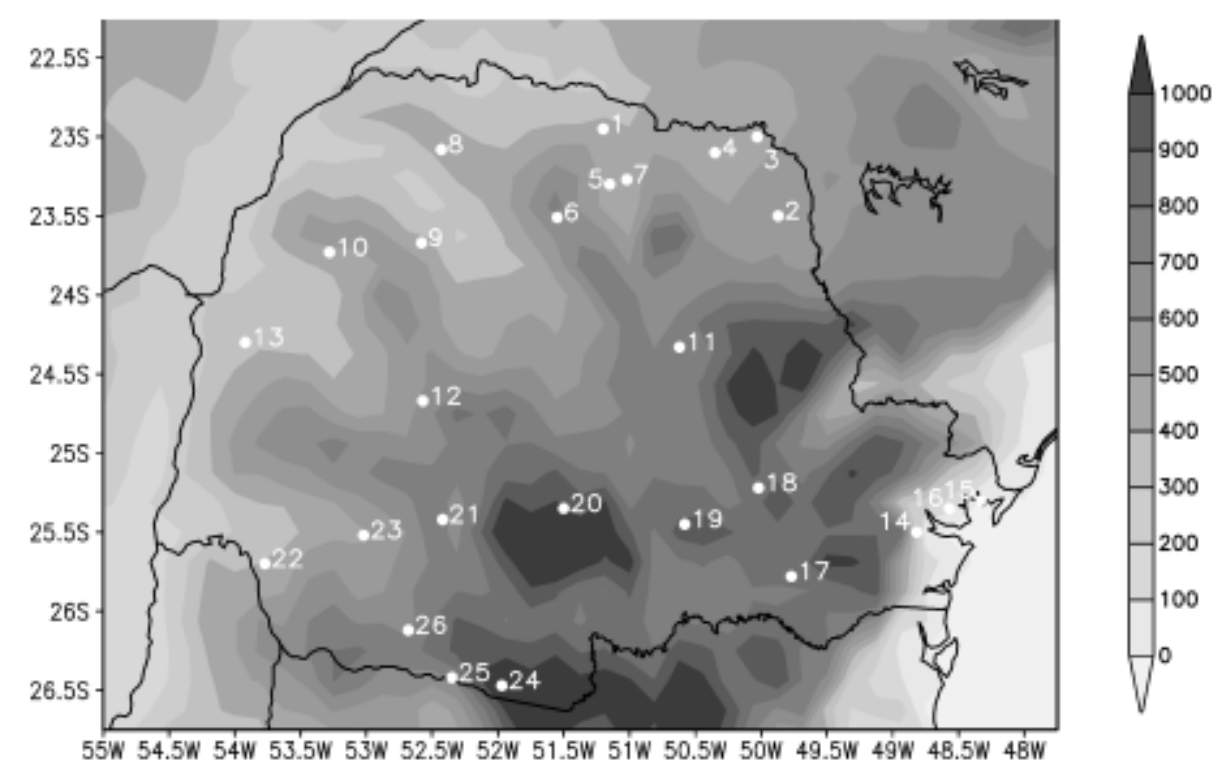

Figura 1 - Localização das estações meteorológicas e topografia da região (sombreado representando o relevo em metros). Identificação: 1- Bela Vista do Paraíso, 2 - Joaquim Távora, 3 - Cambara, 4 - Bandeirantes, 5 - Londrina, 6 - Apucarana, 7 - Ibiporã, 8 - Paranavaí, 9 - Cianorte, 10 - Umuarama, 11- Telêmaco Borba, 12 - Nova Cantu, 13 - Palotina, 14 - Morretes, 15 - Guaraqueçaba, 16 - Antonina, 17 - Lapa, 18 - Ponta Grossa, 19 - Fernandes Pinheiro, 20 - Guarapuava, 21 - Laranjeiras do Sul, 22 - Planalto, 23 - Quedas do Iguaçu, 24 - Palmas, 25 - Clevelândia, 26 - Pato Branco.

Figure 1 - Meteorological stations and topography (grey shading representing relief in meters): 1- Bela Vista do Paraíso, 2 - Joaquim Távora, 3 -Cambara, 4 - Bandeirantes, 5 -Londrina, 6-Apucarana, 7 -Ibiporã, 8 -Paranavaí, 9 -Cianorte, 10-Umuarama, 11 Telêmaco Borba, 12 - Nova Cantu, 13 - Palotina, 14 -Morretes, 15 - Guaraqueçaba, 16 -Antonina, 17 -Lapa, 18 - Ponta Grossa, 19 -Fernandes Pinheiro, 20 - Guarapuava, 21 -Laranjeiras do Sul, 22 -Planalto, 23-Quedas do Iguaçu, 24-Palmas, 25-Clevelândia, 26 - Pato Branco. 


$$
S=\frac{1}{m} \sum_{j=1}^{m}\left[\delta_{j}+\left(1-r_{j}\right)\right] / 2
$$

onde :

$$
\begin{gathered}
\delta=\sqrt{\frac{1}{N} \sum_{i=1}^{N}\left(x_{i}-y_{i}\right)^{2}} \\
r=\frac{\frac{1}{N} \sum_{i=1}^{N}\left(x_{i}-\bar{x}\right) *\left(y_{i}-\bar{y}\right)}{\sigma_{x} \sigma_{y}} ;
\end{gathered}
$$

$\delta$ é a distância Euclidiana, $r$ é o coeficiente de correlação, $m$ é $o$ número de campos comparados, $N$ é o número de casos, $x_{i}$ e $y_{i}$ são as variáveis comparadas e $\sigma_{x}$ e $\sigma_{y}$ são os desvios padrão das variáveis $x$ e $y$, respectivamente. Quanto menor o valor $\delta$, mais alta é a semelhança entre os campos comparados, sendo os campos iguais quando $\delta$ igual a zero. O valor de $r$ varia entre -1 e 1, e os campos são iguais quando $r$ é igual a 1. Portanto, quanto menor o valor de $S$, mais alta é a semelhança entre os dois campos. Se o valor $S$ for igual a zero os campos são iguais.

O procedimento de execução dos análogos é dividido em três etapas: na primeira etapa, por meio da Eq. (1) se executam 160 análogos da temperatura mínima, máxima e chuva acumulada diária nas 26 estações do Paraná com uso das saídas do modelo numérico das previsões de precipitação acumulada 24 horas, umidade especifica no nível $1000 \mathrm{hPa}$ e nebulosidade. Na segunda etapa, com o uso da mesma Eq. (1) e das variáveis restantes da saída do modelo numérico, se extraem os 20 melhores entre os 160 análogos obtidos. Numa terceira etapa, executa-se um grupo de análogos final com o uso da climatologia de freqüência de ocorrência de chuva em cada estação meteorológica. Se, entre os 20 análogos extraídos na estação meteorológica, houver presença de chuva, o grupo de análogos é divido em duas partes, sem chuva e com chuva, e é calculada a porcentagem dos análogos com chuva $\left(P_{0}\right)$ e sem chuva $\left(P_{5}\right)$ no grupo de análogos. Após, os valores

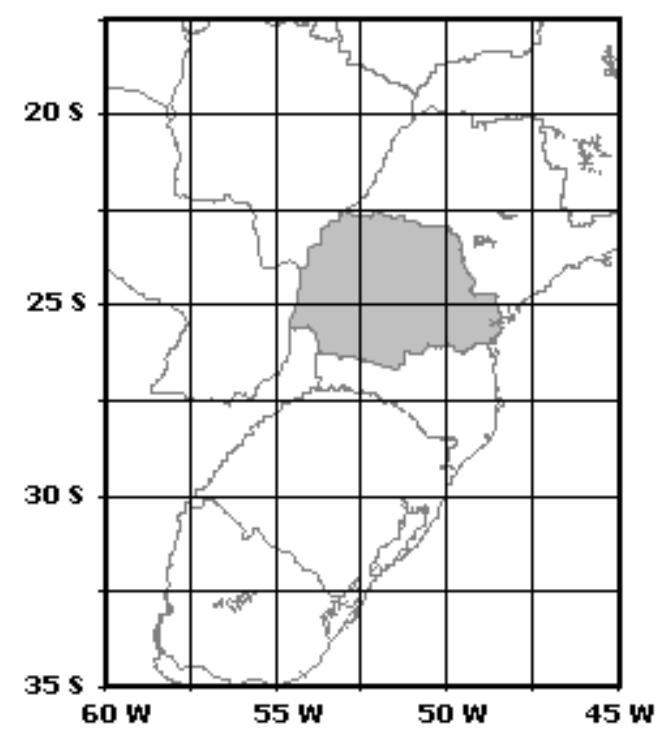

Figura 2 - A região que compreende o setor sul do continente sulamericano e oceanos adjacentes e a grade horizontal do modelo NCEP.

Figure 2 - South sector of South America and surrounding ocean and NCEP model domain grid.

$P_{0}$ e $P_{5}$ são comparados com as probabilidades climáticas de freqüência de ocorrência de dias com chuva e sem chuva, $P_{O C}$ e $P_{S C}$, respectivamente, para cada estação.

$$
q_{o}=\frac{P_{o}}{P_{o c}} \text { e } q_{s}=\frac{P_{s}}{P_{s c}}
$$

Se $q_{o}>q_{s}$ o grupo de análogos final apresenta somente parte dos análogos com chuva e, se $q_{o}<q_{s}$ o grupo final apresenta somente os análogos sem chuva. Finalmente, obtém-se os grupos análogos finais da $\Delta T_{\min }, \Delta T_{\max }$ e chuva acumulada diária para cada uma das estações.

\section{Cálculo da quantidade de chuva e dos valores da temperatura mínima e máxima}

Se $q_{o}>q_{s}$ e o grupo de análogos final na estação apresenta somente ocorrência de chuva, a quantidade de chuva acumulada será calculada através de:

$$
\hat{R}_{i}=\frac{1}{K} \sum_{j=1}^{K} r_{i j}
$$

onde $\hat{R}_{i}$ é a previsão quantidade da chuva na estação 
$i, K$ é o número de análogos com chuva, $r_{i j}$ é a quantidade de chuva no análogo $j$ para estação $i$.

Tanto a temperatura mínima ( $\left.T_{\min }\right)$ quanto a máxima ( $\left.T_{\max }\right)$ são calculadas através de:

$$
\hat{T}_{i}=\frac{1}{K} \sum_{j=1}^{K} \Delta T_{i j}+\bar{T},
$$

onde $\hat{T}_{i}$ é a previsão da temperatura (mínima ou máxima) na estação $i, K$ é o número de análogos no grupo final, $\Delta T_{i j}$ é a anomalia da temperatura no análogo $j$ para estação $i$ e $\bar{T}$ é a norma climática diária da temperatura (mínima ou máxima) na estação $i$.

\section{Normalização}

Como as variáveis do modelo numérico têm diferentes ordens de grandeza, foi aplicado o procedimento de normalização relativa ao desvio padrão:

$$
x_{n i}=\frac{x_{i}-\bar{x}}{\sigma},
$$

em que $x_{n i}$ é o valor normalizado, $x_{i}$ é o valor inicial, $\bar{x}$ é a media aritmética e $\sigma$ é o desvio padrão. Após a normalização, a média torna-se igual a zero e o desvio padrão igual a 1 .

\section{Modelo de componentes cíclicas}

Para suavizar as variações das normas climáticas diárias, que não são monótonas, foi aplicado o modelo de componentes cíclicos:

$$
\hat{\mathrm{Y}}=\sum_{i=1}^{N} A \operatorname{sen}\left(\frac{2 \pi}{L} t+\varphi\right)
$$

onde $A$ é a amplitude, $L$ é o período, $\varphi$ é a fase e $N$ é o número de harmônicos.

\section{Os parâmetros da avaliação das previsões}

A avaliação das previsões das temperaturas mínima e máxima foi feita com a utilização dos seguintes índices de acerto:

Erro médio absoluto:

$$
E M A=\frac{1}{M} \sum_{k=1}^{M}\left|t_{p r}-t_{o b}\right|
$$

Erro médio quadrático:

$$
E M Q=\sqrt{\frac{1}{M} \sum_{k=1}^{M}\left(t_{p r}-t_{o b}\right)^{2}}
$$

onde $t_{p r}$ é a previsão da temperatura, $t_{o b}$ é $a$ temperatura do ar observada e $M$ é o número de estações. Quanto menor o valor do erro médio absoluto e erro médio quadrático, melhor é a qualidade da previsão da temperatura.

A avaliação das previsões de chuva foi feita através dos seguintes índices de acerto:

Acerto total da ocorrência de chuva:

$$
U=\frac{n_{11}-n_{22}}{n_{00}} \cdot 100,
$$

Acerto de ocorrência da presença de chuva:

$$
U_{\text {pres. }}=\frac{n_{11}}{n_{10}} \cdot 100,
$$

Acerto de ocorrência da ausência da chuva:

$$
U_{\text {aus. }}=\frac{n_{22}}{n_{20}} \cdot 100
$$

Índice de acerto em \% das previsões de chuva por intervalos marcados $(\leq 10 \mathrm{~mm})$ :

$$
P_{q}=\frac{n_{1}}{n_{00}} \cdot 100,
$$

Nas equações acima, $n_{11}$ é o número de esta- 


\begin{tabular}{|c|c|c|c|c|c|c|c|c|c|c|c|c|c|}
\hline \multicolumn{14}{|c|}{$\mathrm{P}<0,1 \mathrm{~mm}$} \\
\hline Sub-regãa & I & II & III & IV & $\mathrm{V}$ & VI & V II & V III & IX & $\mathrm{x}$ & $\mathrm{XI}$ & XII & Anual \\
\hline 1 & 23 & 22 & 22 & 17 & 13 & 11 & 13 & 12 & 18 & 20 & 18 & 21 & 209 \\
\hline 2 & 14 & 14 & 11 & 8 & 9 & 8 & 6 & 6 & 9 & 10 & 10 & 13 & 117 \\
\hline 3 & 18 & 16 & 16 & 12 & 11 & 10 & 10 & 9 & 12 & 13 & 13 & 16 & 157 \\
\hline 4 & 16 & 14 & 15 & 12 & 7 & 10 & 5 & 5 & 14 & 16 & 10 & 17 & 141 \\
\hline 5 & 14 & 13 & 12 & 10 & 11 & 11 & 9 & 8 & 10 & 12 & 12 & 13 & 134 \\
\hline 6 & 14 & 14 & 11 & 8 & 10 & 9 & 7 & 7 & 10 & 11 & 11 & 14 & 125 \\
\hline 7 & 14 & 13 & 11 & 8 & 9 & 7 & 6 & 6 & 8 & 10 & 10 & 14 & 115 \\
\hline 8 & 14 & 13 & 12 & 8 & 8 & 6 & 5 & 5 & 9 & 10 & 11 & 14 & 115 \\
\hline \multicolumn{14}{|c|}{$\mathrm{P} \sim 0,1-0,3 \mathrm{~mm}$} \\
\hline Sub-regia ão & I & II & III & IV & $\mathrm{V}$ & VI & V II & V III & IX & $\mathrm{x}$ & $\mathrm{XI}$ & XII & Anual \\
\hline 1 & 1 & 1 & 1 & 2 & 1 & 2 & 2 & 1 & 2 & 1 & 2 & 1 & 16 \\
\hline 2 & 1 & 1 & 1 & 1 & 1 & 0,5 & 0,5 & 0 & 1 & 1 & 1 & 1 & 10 \\
\hline 3 & 1 & 1 & 1 & 1 & 1 & 1 & 1 & 1 & 6 & 1 & 1 & 1 & 9 \\
\hline 4 & 1 & 1 & 1 & 1 & 1 & 1 & 1 & 1 & 1 & 1 & 0 & 1 & 11 \\
\hline 5 & 1 & 1 & 1 & 1 & 1 & 1 & 1 & 1 & 1 & 1 & 1 & 1 & 13 \\
\hline 6 & 3 & 1 & 3 & 2 & 1 & 3 & 1 & 1 & 3 & 0 & 2 & 1 & 21 \\
\hline 7 & 1 & 1 & 1 & 1 & 1 & 1 & 1 & 1 & 0 & 0 & 1 & 1 & 9 \\
\hline 8 & 2 & 2 & 2 & 2 & 3 & 2 & 2 & 2 & 1 & 1 & 2 & 2 & 22 \\
\hline \multicolumn{14}{|c|}{$\mathrm{P} \sim 0,4-10,1 \mathrm{~mm}$} \\
\hline Sub-região & I & II & III & IV & $\mathrm{V}$ & VI & V II & V III & IX & $\mathrm{x}$ & $\mathrm{XI}$ & XII & Anual \\
\hline 1 & 12 & 10 & 11 & 10 & 8 & 7 & 8 & 8 & 11 & 12 & 10 & 11 & 118 \\
\hline 2 & 7 & 7 & 6 & 4 & 4 & 4 & 4 & 4 & 5 & 5 & 5 & 7 & 61 \\
\hline 3 & 11 & 9 & 10 & 8 & 6 & 5 & 5 & 5 & 7 & 7 & 8 & 10 & 90 \\
\hline 4 & 9 & 10 & 9 & 6 & 3 & 3 & 2 & 2 & 4 & 3 & 4 & 6 & 61 \\
\hline 5 & 8 & 7 & 7 & 4 & 5 & 6 & 4 & 4 & 4 & 5 & 4 & 6 & 65 \\
\hline 6 & 7 & 8 & 6 & 4 & 5 & 5 & 3 & 4 & 5 & 5 & 5 & 7 & 63 \\
\hline 7 & 7 & 7 & 6 & 4 & 4 & 3 & 3 & 4 & 4 & 5 & 5 & 6 & 59 \\
\hline 8 & 7 & 7 & 6 & 4 & 4 & 3 & 3 & 3 & 5 & 5 & 5 & 8 & 61 \\
\hline \multicolumn{14}{|c|}{$\mathrm{P} \sim 10,1-20,0 \mathrm{~mm}$} \\
\hline Sub-região & I & II & III & IV & $\mathrm{V}$ & VI & VII & V III & IX & $\mathrm{x}$ & $\mathrm{XI}$ & XII & Anual \\
\hline 1 & 4 & 4 & 4 & 3 & 3 & 1 & 1 & 2 & 3 & 3 & 3 & 4 & 33 \\
\hline 2 & 3 & 3 & 2 & 1 & 2 & 1 & 1 & 1 & 2 & 2 & 2 & 2 & 21 \\
\hline 3 & 3 & 2 & 2 & 1 & 1 & 2 & 2 & 1 & 2 & 2 & 2 & 2 & 23 \\
\hline 4 & 3 & 1 & 2 & 3 & 3 & 1 & 1 & 2 & 7 & 2 & 2 & 3 & 30 \\
\hline 5 & 2 & 3 & 2 & 2 & 2 & 2 & 1 & 1 & 2 & 3 & 3 & 2 & 25 \\
\hline 6 & 3 & 2 & 1 & 1 & 2 & 2 & 1 & 2 & 2 & 2 & 2 & 3 & 22 \\
\hline 7 & 3 & 3 & 2 & 1 & 1 & 1 & 1 & 1 & 2 & 2 & 2 & 3 & 21 \\
\hline 8 & 2 & 2 & 2 & 1 & 1 & 1 & 1 & 1 & 1 & 2 & 2 & 2 & 23 \\
\hline \multicolumn{14}{|c|}{$\mathrm{P} \sim 20,1-30,0 \mathrm{~mm}$} \\
\hline Sub-re gã ão & I & II & III & IV & $\mathrm{V}$ & VI & VII & V III & IX & $\mathrm{x}$ & $\mathrm{XI}$ & XII & Anual \\
\hline 1 & 3 & 2 & 2 & 1 & 1 & 1 & 1 & 1 & 1 & 2 & 2 & 3 & 18 \\
\hline 2 & 2 & 1 & 1 & 1 & 1 & 1 & 1 & 0 & 1 & 1 & 1 & 1 & 11 \\
\hline 3 & 2 & 1 & 1 & 1 & 1 & 1 & 1 & 1 & 1 & 1 & 1 & 2 & 11 \\
\hline 4 & 1 & 1 & 1 & 1 & 1 & 1 & 1 & 1 & 1 & 1 & 2 & 1 & 13 \\
\hline 5 & 1 & 1 & 1 & 1 & 1 & 1 & 1 & 0 & 1 & 1 & 1 & 1 & 12 \\
\hline 6 & 1 & 1 & 1 & 0 & 0 & 1 & 0 & 0 & 0 & 3 & 1 & 2 & 10 \\
\hline 7 & 1 & 1 & 1 & 1 & 1 & 1 & 1 & 0 & 1 & 1 & 1 & 2 & 11 \\
\hline 8 & 1 & 1 & 1 & 1 & 1 & 1 & 1 & 1 & 1 & 2 & 1 & 1 & 12 \\
\hline
\end{tabular}

Tabela 1 - Frequência de ocorrência de precipitação para diferentes intervalos nas 8 sub-regiões do Estado do Paraná.

Table 1 -Frequency of rainfall occurrence for different intervals in the 8 sub-regions of the state of Paraná. 
ções com acerto da presença de chuva, $n_{22}$ é o número de estações com acerto da ausência de chuva, $n_{00}$ é o número total de estações, $n_{10}$ é o número de estações onde ocorreu chuva, $n_{20}$ é o número de estações onde não ocorreu chuva, e $n_{1}$ é o número de estações onde a diferença entre os valores previstos e observados foi menor ou igual a $10 \mathrm{~mm}$.

\section{RESULTADOS E DISCUSSÃO}

\section{Climatologia diária da temperatura mínima, máxima e chuva acumulada}

A análise da freqüência de ocorrência de precipitação e da freqüência de ocorrência dos intervalos de precipitação foi estabelecida por Kim \& Duquia (2000a). Na Tab. 1 estão mostradas as médias mensais e anuais das frequências de ocorrência de precipitação diária acima de $0,1 \mathrm{~mm}$ em 8 estações de apoio (1 - Antonina, 2 - Ponta Grossa, 3 - Palmas, 4-Pato Branco, 5 - Cascavel, 6 - Cianorte, 7 Bela Vista do Paraíso, 8 - Bandedeirantes) que caracterizam as 8 subregiões do estado do Paraná para a precipitação (Kim et al. (2000)). Estas subregiões foram separadas com uso dos dados de chuva acumulada diária nas estações do IAPAR e tem concordância com as condições fisiográficas do estado do Paraná.
O maior número de dias com chuva ocorre no litoral, sendo em média 209 dias por ano (aproximadamente $57 \%$ do total de dias de chuva) e o menor número no norte do estado, com 114 dias (aproximadamente 30\%). A região litorânea representa uma faixa de planície costeira de aproximadamente $20 \mathrm{~km}$, com a parte ocidental da planície costeira estendendo-se até a Serra do Mar com altitudes médias de 850 a 900m (Maack(1981)). Devido a localização próxima do Oceano Atlântico, que proporciona alimentação de umidade constante, a região litorânea apresenta um número maior de dias com chuva.

Observa-se na Tab. 1 que o intervalo de 0,1 $0,3 \mathrm{~mm}$ de precipitação ocorre de 9 a 22 vezes por ano, dependendo da região, enquanto que o intervalo de $0,4-10,0 \mathrm{~mm}$ pode aparecer de 59 a 118 vezes por ano, com máximo nas estações do litoral. Isto significa que cerca de metade dos dias com chuvas ocorre no intervalo $0,4-10,0 \mathrm{~mm}$. O intervalo de 10,1 - 20,0 mm pode ocorrer nas estações do litoral cerca de 33 vezes por ano, sendo que nas outras estações meteorológicas podem ser encontrados cerca de 20 casos na média por ano. O intervalo de 20,1 - 30.0 mm também tem maior freqüência no litoral, cerca de 18 vezes por ano, enquanto que nas outras estações pode ocorrer cerca de 10 a 12 vezes por ano.

A probabilidade de ocorrência dos intervalos de $30,1-40,0 \mathrm{~mm}$ e 40,1 - 50,0 $\mathrm{mm}$ é de 4 a 7 vezes por ano em todas as estações. A mesma probabilidade de ocorrência tem o intervalo $>50 \mathrm{~mm}$,

\begin{tabular}{|c|c|c|c|c|c|c|c|c|c|}
\cline { 2 - 9 } \multicolumn{1}{c|}{} & \multicolumn{10}{c|}{ Sub-região } \\
\hline Intervalo em ${ }^{\circ} \mathrm{C}$ & 1 & 2 & 3 & 4 & 5 & 6 & 7 & 8 & 9 \\
\hline Entre $-1 \mathrm{e}+1$ & 129 & 106 & 108 & 105 & 117 & 135 & 101 & 133 & 113 \\
$+1,1 \mathrm{e}+3$ & 102 & 96 & 92 & 92 & 92 & 101 & 95 & 100 & 87 \\
$-1,1 \mathrm{e}-3$ & 64 & 60 & 56 & 58 & 57 & 61 & 59 & 62 & 57 \\
$>3$ & 32 & 50 & 50 & 53 & 49 & 40 & 51 & 33 & 41 \\
$<-3$ & 39 & 53 & 51 & 57 & 49 & 42 & 59 & 37 & 52 \\
\hline
\end{tabular}

Tabela 2 - Frequência de ocorrência média anual da $\Delta T_{\min }$ para diferentes intervalos nas sub-regiões do Paraná. Table 2 - Frequency of annual mean occurrence of $\Delta T_{\text {min }}$ for different intervals in the 9 sub-regions of the state of Paraná.

\begin{tabular}{|c|c|c|c|c|c|c|c|c|c|}
\cline { 2 - 9 } \multicolumn{1}{c|}{} & \multicolumn{10}{c|}{ Sub-região } \\
\hline Intervalo em ${ }^{\circ} \mathrm{C}$ & 1 & 2 & 3 & 4 & 5 & 6 & 7 & 8 & 9 \\
\hline Entre $-1 \mathrm{e}+1$ & 75 & 84 & 90 & 91 & 89 & 90 & 91 & 98 & 86 \\
$+1,1 \mathrm{e}+3$ & 61 & 75 & 60 & 58 & 58 & 59 & 63 & 58 & 57 \\
$-1,1 \mathrm{e}-3$ & 70 & 61 & 82 & 82 & 81 & 81 & 80 & 87 & 77 \\
$>3$ & 79 & 74 & 65 & 67 & 70 & 70 & 68 & 61 & 79 \\
$<-3$ & 81 & 71 & 64 & 67 & 67 & 65 & 63 & 62 & 67 \\
\hline
\end{tabular}

Tabela 3 - Frequência de ocorrência média anual da $\mathrm{T}_{\max }$ para diferentes intervalos nas sub-regiões do Paraná. Table 3 - Frequency of annual mean occurrence of $T_{\max }$ for different intervals in the 9 sub-regions of the state of Paraná. 
exceto no litoral, onde este intervalo tem freqüência novamente mais alta, cerca de 8 a 9 vezes por ano.

A distribuição de freqüência da ocorrência de chuva e freqüência de ocorrência dos intervalos durante o ano mostrou que, para todas estações do Paraná, o número máximo de dias com chuvas ocorre no verão (janeiro), enquanto que o número mínimo ocorre no inverno, variando entre junho e agosto. A distribuição de freqüência da ocorrência do intervalo 0,1 - 0,3 mm durante o ano não tem regularidade. A distribuição de freqüência de ocorrência dos outros intervalos, em todas as estações, exceto as estações do litoral, tem máximo no verão e mínimo no inverno. Nas estações do litoral existem dois máximos de repetição dos intervalos, em janeiro e outubro, e dois mínimos, em junho e novembro.

A análise da freqüência de ocorrência dos intervalos das anomalias da temperatura mínima $\Delta T_{\min }$ foi feito por Kim \& Duquia (2000b). Na Tab. 2 são mostradas as médias anuais das frequências de ocorrência dos intervalos da temperatura mínima em 9 estações de apoio (1 - Antonina, 2 - Ponta Grossa, 3 - Palmas, 4 - Pato Branco, 5 - Cascavel, 6 Cianorte, 7 Bela Vista do Paraíso, 8-Bandedeirantes, 9 - Cerro Azul) que caracterizam 9 subregiões do estado do Paraná para temperatura mínima (Kim et al. (2000)). Aproximadamente um terço dos dias do ano estão com $\Delta T_{\min }$ no intervalo entre $-1^{\circ} \mathrm{Ce} 1{ }^{\circ} \mathrm{C}$. Cerca de $25 \%$ dos dias do ano $\Delta T_{\min }$ está compreendida na faixa entre $1,1^{\circ} \mathrm{C}$ e $3,0^{\circ} \mathrm{C}$, e $16 \%$ dos dias estão com $\Delta T_{\min }$ no intervalo $-1,1^{\circ} \mathrm{C}$ e $3,0^{\circ} \mathrm{C}$. A repetição do intervalo $>3^{\circ} \mathrm{C}$ é mínima no litoral, aproximadamente $9 \%$ dos dias na média, sendo que nas outras regiões é de $12 \%$. A freqüência de ocorrência do intervalo $<-3{ }^{\circ} \mathrm{C}$ da $\Delta T_{\text {min }}$ é praticamente igual a freqüência do intervalo $>3,0^{\circ} \mathrm{C}$.

$\mathrm{O}$ estudo da distribuição de freqüência de ocorrência do intervalo $-1^{\circ} \mathrm{Ce} 1^{\circ} \mathrm{C}$ da $\Delta T_{\min }$ durante $\mathrm{o}$ ano mostrou que a repetição é mais alta no verão e mais baixa no inverno, com máxima em janeiro ou fevereiro e mínima em junho ou julho. As distribuições de freqüência de ocorrência dos intervalos $-1^{\circ} \mathrm{C} \mathrm{e} \mathrm{-}$ $3^{\circ} \mathrm{Ce} 1^{\circ} \mathrm{Ce} 3^{\circ} \mathrm{C}$ durante o ano são quase uniformes, com pequena variação. Os intervalos $>3^{\circ} \mathrm{C} \mathrm{e}<-$ $3^{\circ} \mathrm{C}$ ocorrem mais freqüentemente no inverno e com menor freqüência no verão e início da primavera, em todas as estações.

As frequências de ocorrência da temperatura máxima $\Delta T_{\max }$ foram analisadas por Kim \& Duquia (2000b). Na Tab. 3 são mostradas as médias anuais das frequências de ocorrência dos intervalos da temperatura máxima em 9 estações de apoio (1 Antonina, 2 - Ponta Grossa, 3 - Palmas, 4 - Pato Branco, 5-Cascavel, 6-Cianorte, 7 Bela Vista do Paraíso, 8 - Telêmaco Borba, 9 - Nova Cantu) que caracterizam 9 subregiões do estado do Paraná para temperatura máxima (Kim et al. (2000)). A distribuição do número de ocorrência dos intervalos da $\Delta T_{\max }$ é quase uniforme. Cerca de $25 \%$ dos dias estão com $\Delta T_{\max }$ no intervalo de $-1{ }^{\circ} \mathrm{C}$ a $1{ }^{\circ} \mathrm{C}$ em todas as estações. Em cerca de $17 \%$ dos dias do ano, $\Delta T_{\max }$ ocorre entre $1,1^{\circ} \mathrm{C}$ e $3,0^{\circ} \mathrm{C}$. Os dias com intervalo de $-1,1^{\circ} \mathrm{C}$ a $-3,0^{\circ} \mathrm{C}$ são cerca de $22 \%$, exceto no

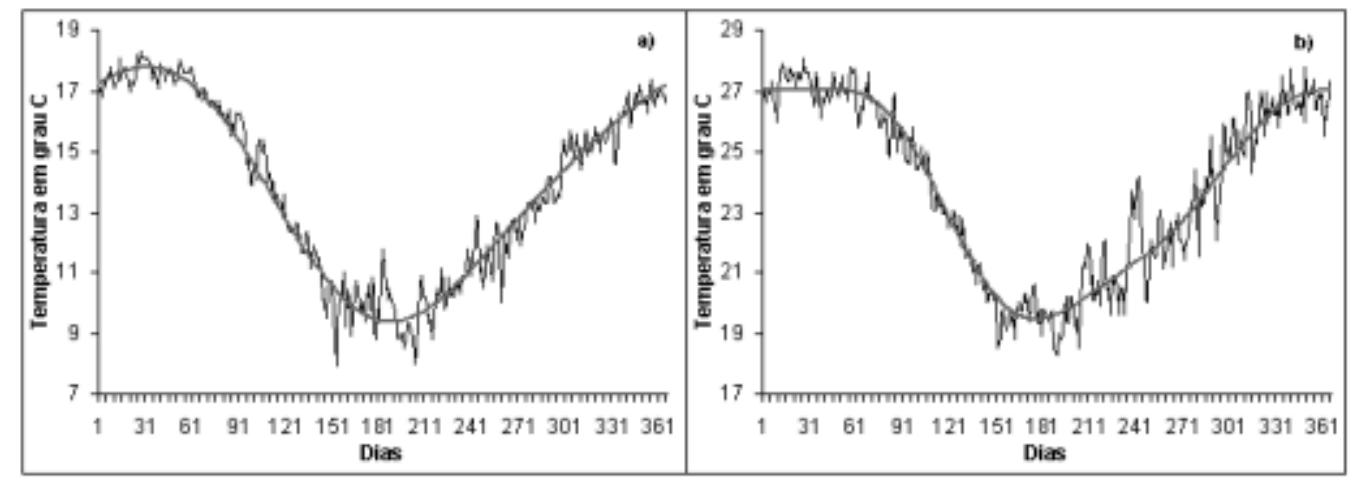

Figura 3 - Normas climáticas diárias da $\mathrm{T}_{\min }$ (a) e $\mathrm{T}_{\max }$ (b) e componentes cíclicas obtidas (negrito) na estação Ponta Grossa.

Figure 3 - Daily climatic norms of $T_{\min }(a)$ and $T_{\max }($ b) and cyclical components (in bold) for the Ponta Grossa station. 
litoral, onde este intervalo pode ocorrer cerca de 18\% dos dias por ano. As freqüências de ocorrência dos intervalos $>3^{\circ} \mathrm{Ce}<-3^{\circ} \mathrm{C}$ são praticamente iguais e cerca de $19 \%$ dos dias.

A distribuição de freqüência de ocorrência do intervalo entre $-1^{\circ} \mathrm{C}$ e $1{ }^{\circ} \mathrm{C}$ durante o ano mostrou que no litoral há três máximos: no verão (janeiro), no outono (abril) e na primavera (novembro) e o número de dias em torno de 7 a 8 . Nas outras estações as distribuições de repetição de dias com intervalo entre $-1^{\circ} \mathrm{C}$ e $1^{\circ} \mathrm{C}$ da $\Delta T_{\max }$ têm distribuição com máximo no verão e mínimo no inverno.

A distribuição de freqüência da ocorrência dos intervalos de $-1^{\circ} \mathrm{Ca}-3^{\circ} \mathrm{C}$ e de $1^{\circ} \mathrm{Ca} 3^{\circ} \mathrm{C}$ da $\Delta T_{\max }$, no litoral, não tem regularidade. Nas outras regiões, apresenta máximo no outono (março) e mínimo na primavera (setembro). Nas estações do litoral, a distribuição de repetição dos intervalos $>3^{\circ} \mathrm{Ce}<$ $3^{\circ} \mathrm{C}$ da é quase uniforme, com aumento insignificante no fim do verão e mínimo no outono. Nas outras estações o maior valor das repetições destes intervalos ocorre no inverno, cerca de 8 a 10 dias por mês, e mínima no verão, com 3 a 4 dias por mês.
Normas climáticas diárias da $\Delta T_{\min }$ e $\Delta T_{\max }$

Foram calculadas as normas climáticas diárias da $\Delta T_{\min }$ e $\Delta T_{\max }$ utilizando todo o período de observação para cada estação meteorológica porém, como o período de observação é curto (na maioria das estações é de cerca de 25 anos), as normas climáticas diárias obtidas não são monótonas. Para suavizar as variações das normas climáticas diárias da $\Delta T_{\min }$ e $\Delta T_{\max }$ foi aplicado o modelo de componentes cíclicas Eq. (8) e foram extraídos não só o ciclo anual, mas uma componente cíclica semianual que tem concordância com Meehl (1991) e Walland \& Simmonds (1999), onde é descrita a existência de uma oscilação semi-anual nas variações da temperatura do ar no Hemisfério Sul. Os conjuntos de componentes cíclicas obtidas descrevem, em geral, mais que $96 \%$ da variância das séries iniciais e os coeficientes de correlação entre as séries iniciais e os conjuntos de componentes cíclicas variam entre 0,95 0,98. Na Fig. 3, como exemplo, são mostradas as variações das normas climáticas diárias da $\Delta T_{\min } \mathrm{e}$

\begin{tabular}{|c|c|c|c|c|c|c|c|c|c|}
\hline & \multicolumn{4}{|c|}{ Inverno } & \multicolumn{5}{|c|}{ Verão } \\
\hline Horas & $U$ & $U_{\text {pres. }}$ & $U_{\text {aus. }}$ & $P_{q}$ & Horas & $U$ & $U_{\text {pres. }}$ & $U_{\text {aus. }}$ & $P_{q}$ \\
\hline 24 & $78 \%$ & $53 \%$ & $82 \%$ & $84 \%$ & 24 & $72 \%$ & $73 \%$ & $62 \%$ & $79 \%$ \\
\hline 48 & $76 \%$ & $50 \%$ & $80 \%$ & $84 \%$ & 48 & $71 \%$ & $71 \%$ & $62 \%$ & $76 \%$ \\
\hline 72 & $75 \%$ & $51 \%$ & $80 \%$ & $81 \%$ & 72 & $68 \%$ & $69 \%$ & $58 \%$ & $76 \%$ \\
\hline 96 & $71 \%$ & $47 \%$ & $78 \%$ & $76 \%$ & 96 & $67 \%$ & $69 \%$ & $56 \%$ & $72 \%$ \\
\hline
\end{tabular}

Tabela 4 - Desempenho das previsões de chuva acumulada diária para 24, 48, 72, 96 horas em média para 26 estações do rede IAPAR do Estado Paraná.

Table 4 - Performance of daily accumulated rain forecasts for 24, 48, 72, 96 hours on average for 26 stations of IAPAR's network in Paraná State.

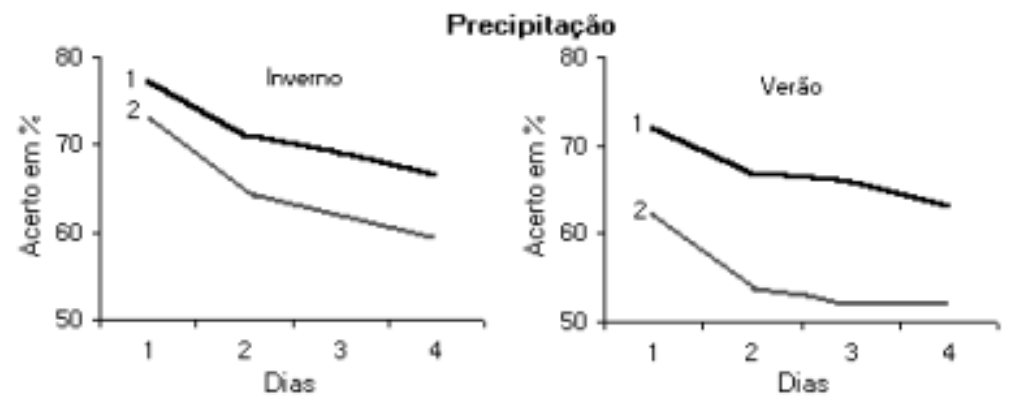

Figura 4 - Acerto das previsões de ocorrência de chuva: 1 - MOS, 2 - persistência.

Figure 4 - Forecast hits of rain occurrence: 1 -MOS, 2 - persistence. 
$\Delta T_{\max }$ e os componentes cíclicos na estação Ponta Grossa.

Como podemos ver na Fig. 3, a componente cíclica suaviza bem as variações das normas climáticas diárias. Os valores suavizados através de componentes cíclicas foram aplicadas no lugar das normas climáticas diárias para o cálculo das anomalias diárias da $\Delta T_{\min }$ e $\Delta T_{\max }$. A análise das normas climáticas diárias da $\Delta T_{\min }$ e $\Delta T_{\max }$ mostrou que as mais altas temperaturas no estado ocorrem no final de janeiro e em fevereiro em todas estações, e as mais baixas temperaturas ocorrem no fim de junho e em julho. Isto significa que o período entre a máxima e a mínima consiste de 5 meses e o período entre a mínima e a máxima, 7 meses, indicando que a temperatura abaixa mais rápido do que sobe.

\section{Previsão da temperatura mínima, máxima e chuva acumulada diária}

As análises feitas anteriormente mostram que somente a distribuição de freqüência da $\Delta T_{\min }$ é quase normal mas a distribuição de freqüência da $\Delta T_{\max }$ é quase uniforme e a distribuição de freqüência de chuva acumulada é $\gamma$. Portanto, para a previsão de $\Delta T_{\max }$ e chuva não é possível aplicar as equações de regressão e por isto foi aplicado o método de grupos análogos.

As equações de regressão múltipla linear estabelecem os erros sistemáticos médios da amostra, mas, como é sabido, os valores dos erros do modelo numérico dependem também de situações atmosféricas. O método de grupos análogos procura, entre os dados históricos da saída do modelo numérico, as previsões mais parecidas com a previsão inicial, e propõem que nas previsões numéricas semelhantes, os erros são semelhantes. O sistema que foi implementado neste trabalho poded ser adaptado, sendo possível adicionar mais dados com o tempo, aumentando o número de casos no banco de dados para executar os análogos.

Os dados da saída do modelo numérico do NCEP e os dados das anomalias de $\Delta T_{\min }, \Delta T_{\max } \mathrm{e}$ chuva acumulada diária foram divididos em dois períodos: de abril a setembro (período do ano frio ou

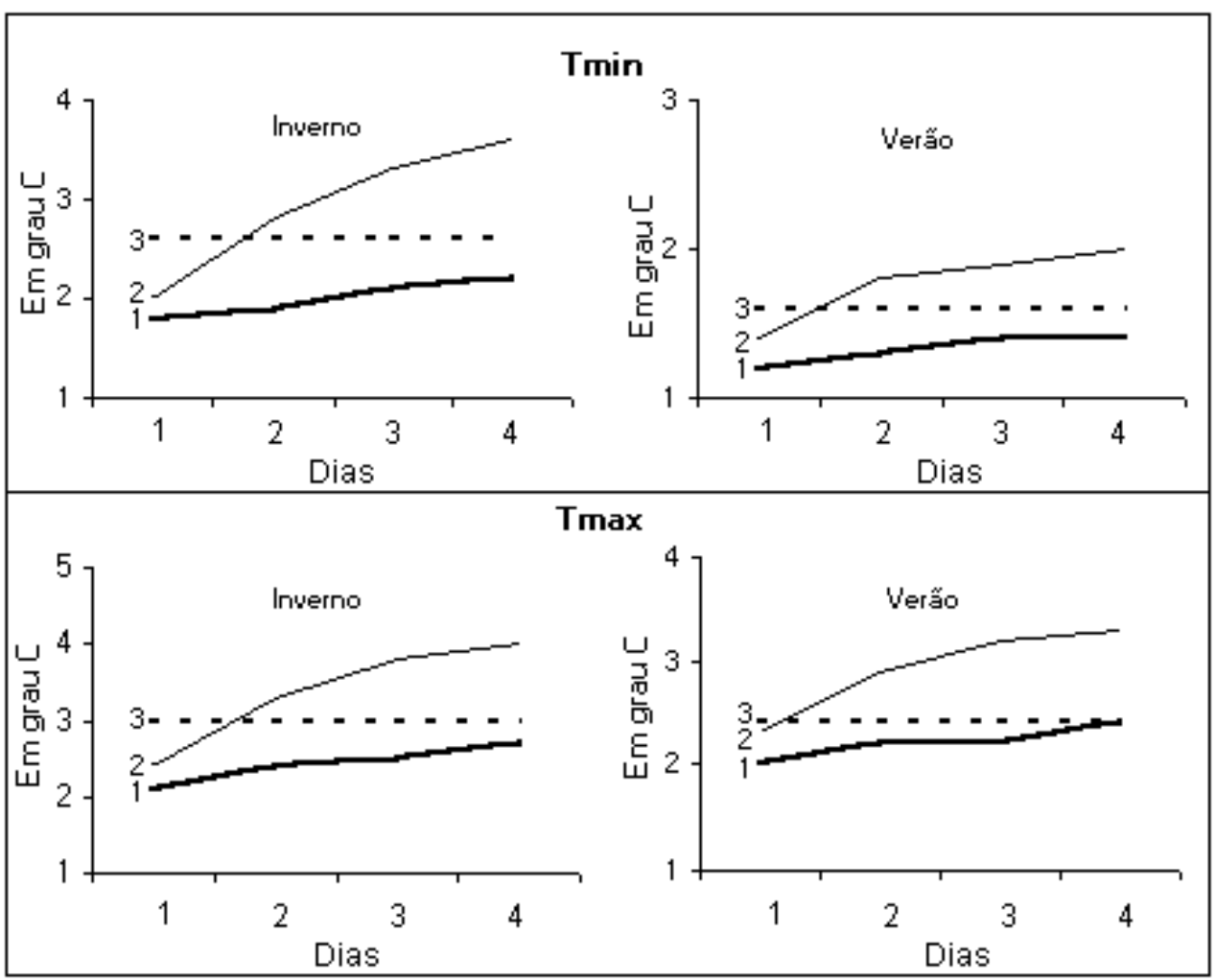

Figura 5 - Erro médio absoluto de previsões até 4 dias: 1 - MOS, 2 - persistência, 3 - climatologia.

Figure 5 - Absolute mean error of forecasts up to 4 days: 1 - MOS, 2 - persistence, 3 -climatology. 
inverno) e de outubro a março (período do ano quente ou verão). Portanto, a amostra de inverno consiste dos dados de abril à setembro dos anos de 1997 à 1999 e a amostra do verão consiste dos dados de outubro à março dos anos de 1997 à 2000.

Os dados da saída do modelo numérico têm diferentes ordens de grandeza: o geopotencial varia entre $10^{1}$ e $10^{4}$, a umidade especifica é de $10^{-3}$, e assim por diante. Para a equalização da ordem de grandeza das diferentes variáveis da saída do modelo numérico foi aplicado o procedimento de normalização (Eq. 7).

Com uso do procedimento dos análogos Eqs. (1), (2), (3) e (4) e cálculo de previsões da $\Delta T_{\min }$, $\Delta T_{\max }$ Eqs. (5) e (6) foram calculadas as previsões para o período de abril de 1997 até março de 2000. As previsões foram calculadas utilizando o método de validação cruzada ("cross validation"), isto é, uma data na amostra de inverno (ou verão) da saída do modelo NCEP e correspondentes dados da $\Delta T_{\text {min }}$, $\Delta T_{\max }$ e chuva diária acumulada para a mesma data foram descartados do banco de dados e estes mesmos dados foram utilizados como dados iniciais, executando-se as previsões da $\Delta T_{\min }, \Delta T_{\max }$ e chuva acumulada diária para 24, 48, 72 e 96 horas. Após o cálculo das previsões, os dados desta data foram devolvidos para o banco de dados e foi executado o mesmo procedimento para as datas seguintes.

\section{Desempenho das previsões.}

As previsões obtidas através de MOS foram avaliadas com uso dos índices de acerto descritos na metodologia. A Tab. 1 mostra os resultados da avaliação das previsões de chuva acumulada diária para $24,48,72,96$ horas.

Os resultados da avaliação das previsões obtidas através do MOS foram comparadas com as previsões de persistência e climatologia. A previsão de persistência significa utilizar a situação de hoje como previsões para os próximos dias. A previsão de climatologia significa utilizar as normas climáticas diárias como previsões para os dias correspondentes. Na fig. 4 são mostradas as avaliações das previsões de chuva obtidas através do MOS e persistência para o inverno e verão. O acerto da previsão de ocorrência de chuva (índice $U$ ) no inverno, para o primeiro dia,

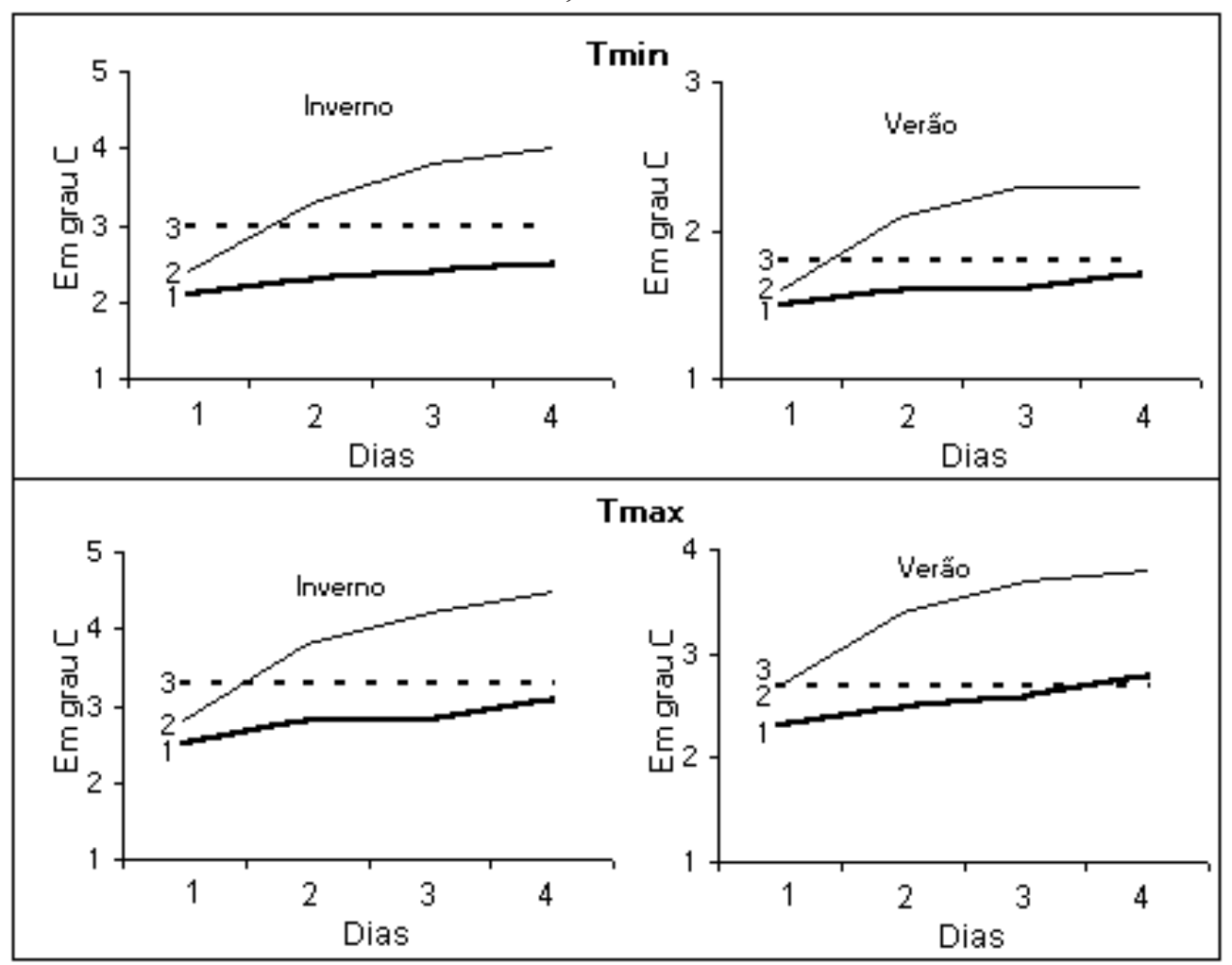

Figura 6 - Erro médio quadrático das previsões até 4 dias: 1 - MOS, 2 - persistência, 3 - climatologia.

Figure 6 - Quadratic mean error of forecasts up to 4 days: 1 -MOS, 2 - persistence, 3 -climatology. 
através do MOS é de $78 \%$, enquanto que a previsão de persistência é de $73 \%$. Para o segundo dia, as previsões do MOS têm acerto de $76 \%$ e as previsões de persistência têm acerto de $65 \%$. Para o terceiro e quarto dias, o MOS acertou $75 \%$ e $71 \%$, respectivamente, e as previsões de persistência tiveram acerto de $61 \%$ e $59 \%$, respectivamente. O acerto das previsões de ocorrência de chuva no verão é mais baixo que no inverno, mas o comportamento dos índices com o aumento da antecedência das previsões é o mesmo. A climatologia da freqüência de ocorrência de chuva mostra que, se sempre prevermos ausência de chuva, receberemos um índice $U$, igual a $67 \%$ para o inverno e $54 \%$ para o verão. Portanto, as previsões de ocorrência de chuva através do MOS têm vantagem diante das previsões de persistência e da climatologia.

Na Tab. 4 são mostrados os valores dos índices de acerto $U_{\text {pres. }}, U_{\text {aus. }}$ e $P_{q}$. No inverno, o índice de acerto $U_{\text {aus. }}$ tem valores mais altos que no verão, variando de $82 \%$ para o primeiro dia até $78 \%$ para o quarto dia, enquanto que no verão variam de $62 \%$ até $56 \%$. Contrariamente, o índice $U_{\text {pres. }}$ no verão é mais alto que no inverno, variando entre $73 \%$ e $69 \%$ no verão e entre 53 e $47 \%$ no inverno. Os valores do índice $P_{q}$ para as previsões do inverno variam entre $84 \%$ e $76 \%$ e para o verão variam entre $79 \%$ e $72 \%$.

Na Fig. 5 são mostradas as variações de EMA das previsões do MOS, persistência e climatologia da $\Delta T_{\min }, \Delta T_{\max }$ em função do tempo. Como podemos ver, o EMA da $\Delta T_{\min }$ de climatologia não muda durante o tempo, enquanto o do MOS e da persistência cresce com o aumento da antecedência. $\mathrm{O}$ valor do EMA de persistência para o primeiro dia é menor que o valor do EMA da climatologia, mas a partir do segundo dia, o valor de EMA de persistência é mais alto e cresce com o tempo. Os EMAs do MOS no inverno variam de 1,8 no primeiro dia até 2,2 no quarto dia, enquanto que no verão estes valores variam entre 1,2 e 1,4 e estão abaixo do EMA de climatologia (2,6 no inverno e 1,6 no verão) e persistência (variando entre 2,0 e 3,6 no inverno e 1,4 e 2,0 no verão) para ambas as estações do ano. O mesmo comportamento pode ser observado nas variações do EMA da $\Delta T_{\max }$, que também são mostradas na Fig.5. Porém, os valores do EMA da $\Delta T_{\max }$ são mais altos que para $\Delta T_{\min }$, variando entre 2,1 e 2,7 no inverno e entre 2,0 e 2,4 no verão. No mesmo tempo, o EMA da persistência varia entre 2,3 e 3,9 no inverno e entre 2,3 e 3,3 no verão, enquanto o valor do EMA da climatologia da $\Delta T_{\max }$ é constante e igual a 3,0 no inverno e 2,4 no verão.

As variações do índice de acerto EMQ de $\Delta T_{\min }$ e $\Delta T_{\max }$ através do MOS, da persistência e da climatologia são mostrados na Fig. 6. O EMQ da $\Delta T_{\text {min }}$ de climatologia também não muda durante o tempo e são iguais a 3,0 no inverno e 1,8 no verão. Para a $\Delta T_{\max }$, os valores do EMQ são iguais a 1,8 no inverno e 2,7 no verão. O valor do EMQ da persistência no primeira dia é menor que o valor do EMQ da climatologia, mas cresce rápido durante o tempo e a partir do segundo dia são maiores que o EMQ da climatologia. Verifica-se na Fig. 6 que os valores do EMQ do MOS para $\Delta T_{\min }$ são menores que os valores do EMQ da persistência e climatologia para as previsões até 4 dias. Mas para $\Delta T_{\max }$ no quarto dia do período de verão os valores do EMQ do MOS e da climatologia são iguais.

A qualidade das previsões do MOS da $\Delta T_{\text {min }}$, em média, tem vantagem diante das previsões da persistência e climatologia para todo o ano. A qualidade das previsões do MOS da $\Delta T_{\max }$, em média, é mais alta que as previsões de persistência e climatologia no inverno. No verão esta vantagem dura até 3 dias de previsão, sendo que no quarto dia os valores do EMA e EMQ do MOS e climatologia são iguais.

\section{CONCLUSÃO}

Foi feito um estudo de climatologia diária das $\Delta T_{\min }$ e $\Delta T_{\max }$ e chuva acumulada para o estado do Paraná. Na maior parte das estações do Estado, cerca de 1/3 dos dias do ano há ocorrência de chuva, exceto no litoral, onde as chuvas são mais freqüentes. 
Cerca de metade do número de chuvas está no intervalo $0.4-10 \mathrm{~mm}$. A distribuição de freqüência de ocorrência de chuva durante o ano mostrou que tem máxima no verão e mínima no inverno em todas estações do Paraná mas, ao mesmo tempo, a amplitude das variações são diferentes. As distribuições de freqüência de ocorrência dos intervalos durante o ano mostrou que a partir do intervalo 0,4 -10,0 mm existem máxima no verão e mínima no inverno para todas as estações do Paraná.

A distribuição de freqüência da ocorrência da $\Delta T_{\min }$ é quase normal. A freqüência de ocorrência do intervalo - 1 e $1^{\circ} \mathrm{C}$ da $\Delta T_{\min }$ é mais alta no verão do que no inverno. As freqüências de ocorrências dos intervalos de $-1,1 \mathrm{a}-3{ }^{\circ} \mathrm{Ce}$ de 1,1 a $3{ }^{\circ} \mathrm{C}$ praticamente são as mesmas em todas as estações do ano e as freqüências de ocorrências dos intervalos $>3^{\circ} \mathrm{Ce}<$ $-3^{\circ} \mathrm{C}$ são mais altas no inverno do que no verão. As freqüências de ocorrências dos intervalos da $\Delta T_{\max }$ nas estações do Paraná são quase uniformes. As freqüências de ocorrências dos intervalos de $-1^{\circ} \mathrm{Ca}$ $1^{\circ} \mathrm{C}$, de 1,1 a $3^{\circ} \mathrm{C}$ e de $-1,1$ a $-3^{\circ}$ da $\Delta T_{\max }$ tem mínima no fim do inverno e início da primavera e máxima no verão e início do outono e o contrário para os intervalos $>3^{\circ} \mathrm{C} \mathrm{e}<-3^{\circ} \mathrm{C}$

Foi implementado o MOS para previsão da $\Delta T_{\min }, \Delta T_{\max }$, ocorrência e quantidade de chuva até 4 dias. O MOS foi usado com o método de grupos análogos, onde foi aplicado um parâmetro de semelhança complexo que consiste como critérios de semelhança a distância Euclidiana e o coeficiente de correlação. Como preditores foram aplicadas as saídas do modelo numérico do NCEP para 36, 60 84 e 108 horas e como preditantes as $\Delta T_{\min }, \Delta T_{\max }$ e chuva acumulada diária nas 26 estações do estado do Paraná.

Usando o método de validação cruzada, as previsões do MOS para até 4 dias foram avaliadas para o período de abril de 1997 à março de 2000. A ocorrência de chuva é prevista com acerto de $78 \%$, $76 \%, 75 \%$ e $71 \%$ para 1 a 4 dias, respectivamente, para o inverno, e $72 \%, 71 \%, 68 \%$ e $67 \%$ para o verão. Os erros médios absolutos da previsão da
$\Delta T_{\min }$ são $1,8,1,9,2,1$ e 2,2 para 1 a 4 dias, respectivamente, para o inverno, e 1,2, 1,3, 1,4 e 1,4 para o verão. A $\Delta T_{\max }$ é prevista com erros médios absolutos de 2,1, 2,4, 2,5 e 2,7 para 1 a 4 dias, respectivamente, para o inverno, e 2,0, 2,2, 2,2 e 2,4 para o verão. As comparações das previsões do MOS para o mesmo período mostrou a boa vantagem das previsões do MOS diante das previsões da persistência e da climatologia .

\section{REFERÊNCIAS}

Azcarraga, R. \& Ballester, A.J.G., 1991. Statistical system for forecasting in Spain. In Programme on Short- and Medium-Range Weather Prediction Research, H. R. Glahn, A.H. Murphy, L.J. Wilson and J.S. Jensenius, eds., WM/TD No. 421. World Meteorological Organization, Geneva, XX23-25.

Brunet, N., Verret, R. \& Yacowar, N., 1988. An objective comparison of model output statistics and "perfect prog" systems in producing numerical weather element forecasts. Weather and Forecasting, 3: 273-283.

Carter, G.M., Dallavalle, J.P. \& Glahn, H.R., 1989. Statistical forecasts based on the National Meteorological Center's numerical weather prediction system. Weather and Forecasting, 4: 401-412.

Conte, M., de Simone, C. \& Finizo, C., 1980. Postprocessing of numerical models: Forecasting the maximum temperature at Milano Linate. Rev. Meteorol. Aeronaut., 40: 247-265.

Erickson, M.C., Bower, J.B., Dagostaro, V.J., Dallavalle, J.P., Jacks, E., Jensenius, J.S. \& Su, J.C., 1991. Evaluation the impact of RAFS changes on the NGM-based MOS guidance. Weather and Forecasting, 6: 142-147.

Esterle, G.R., 1992. Adaptive, Self-learning statistical interpretation system for the Central Asian region. Annals Geophysical 10. p. 924-929.

Francis, P.E., Day, A.P. \& Davis, G.P., 1982. Automated temperature forecasting, and application of Model Output Statistics to the Meteorological Office numerical weather prediction model. Meteorol. Mag., 111: 73-87. 
Jacks, E., Bower, B., Dagostaro, V.J., Dallavalle, P.J., Erickson, M.C. \& Su, J.C., 1989. New NGM-Based MOS Guidance for Maximum/ Minimum Temperature, Probability of Precipitation, Cloud Amount, and Surface Wind. Weather and Foresting, November 7-13, vol. 5, p.128-138.

Kim, I.S., Quadro, M.F.L. de \& Marengo, J., 1998. A Aplicação do "Model Output Statistics" para previsão da temperatura mínima no Sul e Sudeste do Brasil. X Congresso Brasileiro de Meteorologia. Brasília.

Kim, I.S. \& Duquia C.G., 2000a. Um Estudo Climático das Variações de Precipitação Diária no Estado do Paraná. XI Congresso Brasileiro de Meteorologia, Rio de Janeiro.

Kim, I.S. \& Duquia C.G., 2000b. Um Estudo Climático das Variações de Temperatura Mínima e Máxima Diária no Estado do Paraná. XI Congresso Brasileiro de Meteorologia, Rio de Janeiro.

Kim, I.S.; Duquia C.G. \& Calvetti, L., 2000. Regionalização do Estado do Paraná com a utilização dos Campos de Precipitação, Temperatura Mínima e Máxima. XI Congresso Brasileiro de Meteorologia, Rio de Janeiro.

Lemcke, C. \& Kruizinga, S., 1988. Model output statistics forecast: Three years of operational experience in the Netherlands. Mon. Weather Rev., 116: 1077-1090.

Lu, R., 1991. The application on NWP products and progress of interpretation techniques in China. In Programme on Short- and Medium-Range
Weather Prediction Research, H. R. Glahn, A.H. Murphy, L.J. Wilson and J.S. Jensenius, eds., WM/TD No. 421. World Meteorological Organization, Geneva, XX19-22.

Maack, R., 1981. Geografia Física do Estado do Paraná, Rio de Janeiro, 450p.

Meehl, G.A., 1991. A reexamination of the mechanism of the semiannual oscillation in the Southern Hemisphere. J. Climate, 4, 911-926.

Tapp R.G., Woodcock, F. \& Mills G.A., 1986. The Application of Model Output Statistics to Precipitation Prediction in Australia. Monthly Weather Review, vol. 114: N 1, p. 50-61.

Vasiliev, P.P., 1991. Medium-range air temperature and precipitation forecasting over Eurasia. Meteorologia i Gidrologia, N 2, p. 13-23.

Vislocky R.L. \& Fritsch J.M., 1995. Improved Model Output Statistics Forecasts through Model Consensus. Bull. Of the Am. Met. Soc. Vol. 76, N 9, P. 1157-1164.

Vislocky R.L. \& Fritsch J.M., 1997. An automated, Observation-Based System for Short-Term Prediction of Ceiling and Visibility. Weather and Forecasting, Vol. 12: N 3 P. 31-43.

Walland, D. \& Simmonds, I., 1999. Baroclinicity, Meridional Temperature Gradients, and the Southern Semiannual Oscillation. J. Climate, 12, 3376-3382.

Wilks, D.S., 1990. Probabilistic quantitative precipitation forecast derived from PoPs and conditional precipitation amount climatologies. Mon. Wea. Rev., 118: p. 874-882.

\section{EXPANDED ABSTRACT}

Daily climatology of minimum and maximum temperature anomalies $\left(\Delta T_{\min }, \Delta T_{\max }\right)$ and also accumulated precipitation, for Paraná State were studied in this work. Rainfall occurs in about $1 / 3$ of the total days of the year, for most of the stations, except on the coast, where rains more frequently. Frequency of rainfall occurrences for intervals 0.1 $0.3 \mathrm{~mm}, 0.4-10 \mathrm{~mm}, 10.1-20 \mathrm{~mm}, 20.1-30 \mathrm{~mm}, 30.1-40 \mathrm{~mm}$, $40.1-50 \mathrm{~mm}$ and $>50 \mathrm{~mm}$ was analyzed in this study. About half of the rainfall occurrence is in the interval 0.4 to $10 \mathrm{~mm}$. Frequency distribution of rainfall occurrence during the year showed a maximum in the summer and minimum in the winter for all the stations but, at the same time, amplitudes of variations are different. Frequency distribution of rainfall intervals during the year showed that for the interval 0.4 to $10 \mathrm{~mm}$ there is a maximum in the summer and a minimum in the winter for all the stations. Frequency of occurrence of $\Delta T_{\min }$ lying in the interval $-1^{\circ} \mathrm{C}$ to $1^{\circ} \mathrm{C}$ is higher in the summer than in the winter. Frequency of occurrence of intervals $-3^{\circ} \mathrm{C}$ to $-1.1^{\circ} \mathrm{C}$ and $1.1^{\circ} \mathrm{C}$ to $3^{\circ} \mathrm{C}$ is practically the same for all seasons, whereas 
frequency of occurrence of the intervals $>3^{\circ} \mathrm{C}$ and $<$ $3^{\circ} \mathrm{C}$ is higher in the winter. Frequency distribution of $\Delta T_{\min }$ occurrences for all stations is almost normal. Frequency distribution of $\Delta T_{\max }$ occurrences for the data is almost uniform. Frequency of occurrence of intervals $-1^{\circ} \mathrm{C}$ to $1^{\circ} \mathrm{C}, 1.1^{\circ} \mathrm{C}$ to $3^{\circ} \mathrm{C}$ and $-3^{\circ} \mathrm{C}$ to $1.1^{\circ} \mathrm{C}$ for $\Delta T_{\max }$ has minimum by the end of the winter and beginning of spring, and maximum during the summer and beginning of autumn, and the opposite for the intervals $>3^{\circ} \mathrm{C}$ and $<-3^{\circ} \mathrm{C}$. A Model Output Statistics (MOS) was applied for the forecast of up to 4 days of minimum and maximum temperatures, rainfall occurrence and quantity. An analogs group method was used in the model with a complex analogy parameter including Euclidean distance and correlation coefficient. NCEP numerical model outputs for $36,60,84$ and 108 hours forecasts were used as predictors and minimum and maximum temperature, and daily rainfall accumulations for 26 stations in Paraná were predictants. Using a cross validation method, MOS forecasts for up to 4 days were evaluated for the period of April 1997 to March 2000. Rainfall occurrence is predicted correctly $78 \%, 76 \%$, $75 \%, 71 \%$ for 1 to 4 days, respectively, during the winter, and $72 \%, 71 \%, 68 \%, 67 \%$ for 1 to 4 days, respectively, during the summer. Mean absolute errors for minimum temperature are 1.8, 1.9, 2.1 and 2.2 for 1 to 4 days, respectively, during the winter, and 1.2, $1.3,1.4,1.4$ for 1 to 4 days, respectively, during the summer. Maximum temperature is predicted with mean absolute errors of $2.1,2.4,2.5,2.7$ for 1 to 4 days, respectively, during the winter, and 2.0, 2.2, 2.2, 2.4 for 1 to 4 days, respectively, during the summer. Comparisons of MOS forecasts with persistence and climatology for the same period showed a significant advantage in using MOS forecasts.

\section{UnB - University of Brasilia Institute of Geosciences}

\section{Laboratory of Applied Geophysics}

Offers graduate and undergraduate courses associated with the Geology Program of the UnB. The laboratory is responsible for the area of specialization in Applied Geophysics belonging to the Graduate Program in Geology and leading to MSc's and PhD's degrees. The main areas of research are: airbone geophysics applied to geological mapping, mineral and hidrocarbon exploration, ground-water and, environmental studies; ground geophysics applied to mineral exploration, groundwater, environmental, forensic, and geotechnical studies; interpretation (modeling, inversion, and statistical analysis) and integration methods of geophysical data.

Visit: http://www.unb.br/ig/labo/lga. Contact: labgeo@unb.br

\section{CONSELHO NACIONAL DE DESENVOLVIMENTO CIENTÍFICO E TECNOLÓGICO - CNPq Diretório dos Grupos de Pesquisa no Brasil}

O Diretório dos Grupos de Pesquisa no Brasil é uma base de dados desenvolvida no $\mathrm{CNPq}$ desde 1992. Ela contém informações sobre os grupos de pesquisa em atividade no país. Almeja, portanto, um caráter censitário. As informações disponíveis 
indicam que na versão 4.0 o Diretório conseguiu alcançar uma cobertura acima de $80 \%$ dos grupos de pesquisa em atividade no país. As informações constantes na base dizem respeito aos recursos humanos constituintes dos grupos, às linhas de pesquisa em andamento, às especialidades do conhecimento, aos setores de atividade envolvidos, aos cursos de mestrado e doutorado com os quais o grupo interage e à produção científica e tecnológica nos três anos imediatamente anteriores à época da coleta dos dados. Dessa forma, cada grupo é situado no espaço e no tempo.

Os grupos de pesquisa inventariados estão localizados em universidades, instituições isoladas de ensino superior, institutos de pesquisa científica, institutos tecnológicos, laboratórios de pesquisa e desenvolvimento de empresas estatais ou ex-estatais, e em algumas organizações não-governamentais com atuação em pesquisa.

Sua primeira versão (1.0) apresentou informações referentes ao segundo semestre de 1993, sendo a produção científica e tecnológica correspondente ao triênio 1990-1992. A versão 2.0 apresentou informações referentes ao segundo semestre de 1995, e a produção correspondeu ao biênio 1993-1994. A versão passada (3.0) apresentou informações referentes ao segundo semestre de 1997 e à produção do período de $1^{\circ}$ de janeiro de 1995 a 30 de junho de 1997. A versão atual (4.1) substitui a versão 4.0. Ela apresenta informações sobre os grupos coletados no primeiro semestre de 2000 e à produção C\&T do período de janeiro de 1997 a dezembro de 2000. Essa produção, relativa aos pesquisadores e estudantes cadastrados no Diretório no primeiro semestre de 2000, é a existente na base de Currículo Lattes do CNPq em 01 de junho de 2001 (a versão 4.1 atualizou apenas os dados da produção $C$, T\&A da versão 4.0, que abrangia a produção do triênio 1997-1999 existente no CNPq em 10 de novembro de 2000). Desde a versão 3.0, de 1997, o Diretório é capaz de descrever com precisão os limites e o perfil geral da atividade científico-tecnológica no Brasil. Igualmente, é capaz de fornecer aos interessados uma grande massa de informação, bastante diversificada, sobre detalhes de quem realiza as atividades, como e onde se realizam e sobre 0 quê. Nesta versão que apresentamos (4.1), a estas capacidades agregam-se outras. Em primeiro lugar, a base de dados relativa à versão 4.0 (2000) foi atualizada no que se refere à produção científica, tecnológica e artística. Essa atualização foi feita através da migração das informações do Currículo Lattes, existentes no CNPq em 01/06/2001. Em segundo lugar, foi implantado o recurso de registrar no tempo a evolução dos grupos (no sentido de continuidade), por meio do desenvolvimento de modelos de Data Warehouse. Isso situa o objetivo de estabelecer uma memória da atividade de pesquisa em outro patamar. Em terceiro lugar, a atual versão, pelo desenvolvimento da Plataforma Lattes, integrou-se, em terreno mais sólido, às bases de dados da Capes, ao Currículo Lattes e ao importante sistema gerencial do $\mathrm{CNPq}$ (Sigef), que, entre outras finalidades, realiza a gerência dos bolsistas de produtividade em pesquisa. E, em quarto lugar, estão sendo desenvolvidas ferramentas capazes de instrumentar a agência e a comunidade científica com o objetivo de fomentar e gerir a atividade de pesquisa. A principal delas é o algoritmo de hierarquização qualitativa dos grupos de pesquisa. 